\title{
Anaplastic Large Cell Lymphoma, ALK-Negative Presenting in the Rectum: A Case Report and Review of the Literature
}

\author{
Komal Galani' ${ }^{1}$, Suryakanth R. Gurudu' ${ }^{2}$, Longwen Chen $^{3}$, Katalin Kelemen ${ }^{3}$ \\ ${ }^{1}$ Super Religare Laboratories, Nagpur, India; ${ }^{2}$ Department of Gastroenterology, Mayo Clinic, Phoenix, USA; ${ }^{3}$ Department of Labo- \\ ratory Medicine and Pathology, Mayo Clinic, Phoenix, USA. \\ Email: drkomalg@gmail.com
}

Received September $27^{\text {th }}, 2012$; revised October $27^{\text {th }}, 2012$; accepted November $17^{\text {th }}, 2012$

\begin{abstract}
ALK-negative anaplastic large cell lymphoma (ALCL, ALK-) is a CD30+ T-cell neoplasm composed of large lymphoid cells with abundant cytoplasm and pleomorphic nuclei that lacks expression of ALK protein. We describe a case of ALCL, ALK - with primary involvement of the rectum in a 37-year-old man, where the original diagnosis was established based on a colonoscopic biopsy. T-cell lymphomas are rare in the colorectal area and besides ALCL, their differential diagnosis includes enteropathic T-cell lymphoma, peripheral T-cell lymphoma, not otherwise specified, NK/T cell lymphoma, or NK-cell enteropathy. In addition, syncytial variant of classical Hodgkin lymphoma or a pleomorphic CD30-positive diffuse large B-cell lymphoma should also be ruled out. We discuss pitfalls of the differential diagnosis and review the literature of anaplastic large cell lymphoma in the gastrointestinal tract. Correct diagnosis of ALCL in the colon is important to avoid a colorectal surgery for an assumed adenocarcinoma, and to open the possibility for lymphoma-directed chemotherapy.
\end{abstract}

Keywords: Anaplastic Large Cell Lymphoma; CD30+ Lymphoma; Gastrointestinal Lymphoma

\section{Introduction}

Anaplastic large cell lymphoma (ALCL) is a CD30positive neoplasm of T-cell or null-cell lineage with characteristic clinicopathologic features, and accounts for $3 \%$ of all adult non-Hodgkin lymphomas (NHL). Two subsets of systemic ALCL are currently recognized in the World Health Organization (WHO) classification. One subset expresses the oncogenic protein anaplastic lymphoma kinase (ALK) as a result of either $t(2 ; 5)(\mathrm{p} 23$; q35) translocation or variant alk rearrangement. The second subset includes ALK-negative (ALK-) tumors that cannot be distinguished from ALCL, ALK-positive $(\mathrm{ALK}+)$ based on morphological features and also express CD30 [1]. The provisional ALK - ALCL category was formed to reflect distinguishing clinical data, including the advanced median age and more aggressive clinical course of cases of ALK-negative ALCL. We describe a unique case of ALK- ALCL presenting as an incidental small rectal ulcer.

\section{Case Report}

A 37-year-old male presented with a 7-month history of episodic fevers, night sweats, joint pain and skin rash. He also complained of recent onset nausea, vomiting and left upper quadrant abdominal pain. His medical history included a left nephrectomy for renal cell carcinoma six years ago. Physical examination showed mild cervical lymphadenopathy and splenomegaly. His complete blood count and metabolic panel were within normal limits. Numerous rheumatologic studies were negative. Computed Tomography (CT) and Magnetic Resonance Imaging (MRI) scans of the abdomen revealed fat stranding and inflammatory changes with mesenteric edema extending to the left upper quadrant, splenomegaly of 15 $\mathrm{cm}$ in greatest dimension with no focal lesions, and indeterminate retroperitoneal lymph nodes. Endoscopic examination of the stomach and duodenum were unremarkable. Colonoscopy showed multiple mediummouthed diverticula in the entire colon and a small aphthous-appearing ulcer was noticed in the rectum (Figure 1) which was biopsied. Microscopic examination revealed ulcerated rectal mucosa and a heterogeneous infiltrate of large pleomorphic cells with abundant pale cytoplasm, vesicular chromatin and prominent nucleoli (Figure 2). The large cells were seen in a background of small lymphocytes, neutrophils and frequent apoptotic cells. Immunohistochemistry was performed with the 


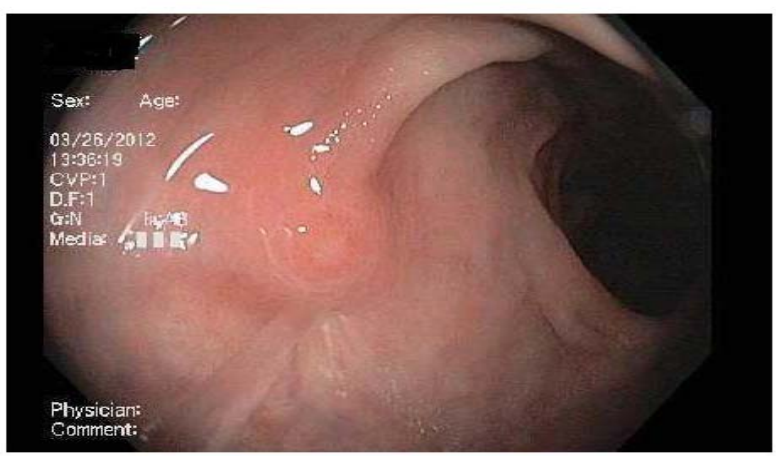

Figure 1. Colonoscopic image of a single aphthous-appearing ulcer detected in the rectum. Biopsy was performed for histology with a cold forceps.

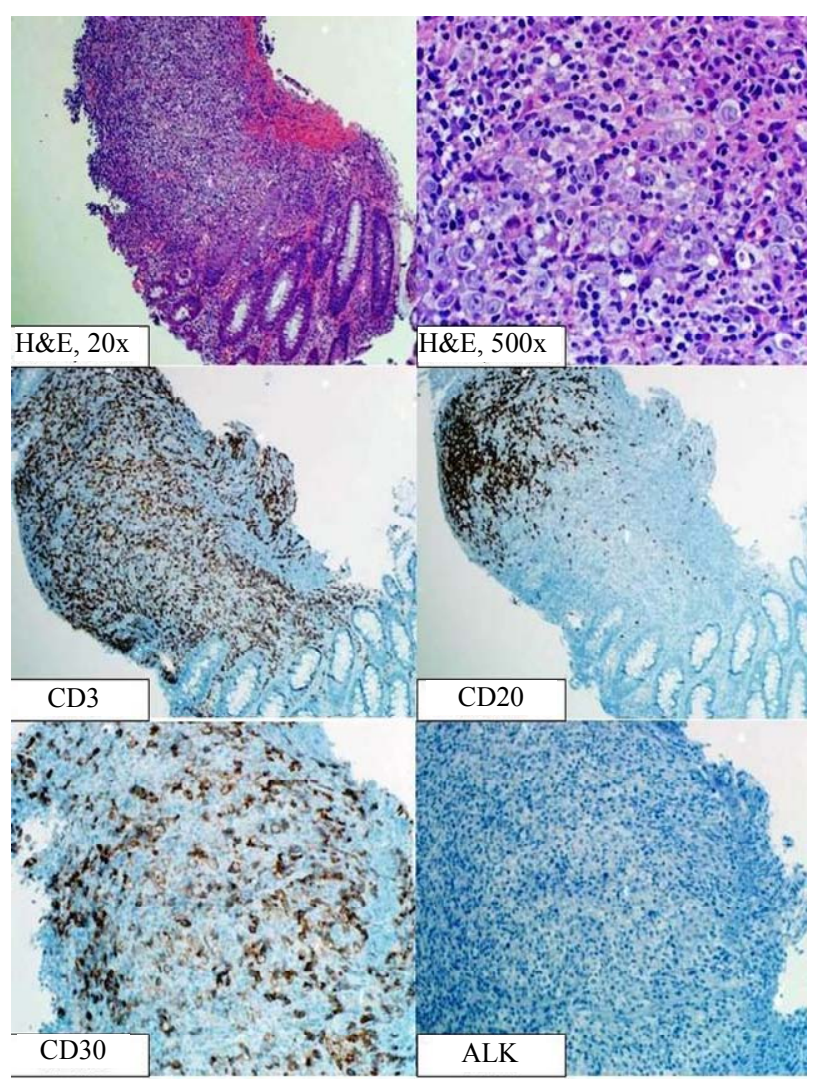

Figure 2. Histologic and immunophenotypic findings of the rectal biopsy. The biopsy shows colonic mucosa with ulceration and proliferation of large pleomorphic cells with abundant pink cytoplasm and visible-to-prominent nucleoli. The large cells are admixed with a background of small lymphocytes, numerous neutrophils and frequent apoptotic figures. Immunostains show the large cells are negative for CD3 and CD20, and are strongly positive with CD30, with a membranous and Golgi-zone pattern. The large cells are negative for ALK. The small lymphocytes in the background are predominantly CD3 positive T-cells.

following antibodies: mouse anti-ALK1, rabbit anti-CD3, rabbit anti-CD4, rabbit anti-CD5, mouse anti-CD15, mouse anti-CD20, mouse anti-CD30, mouse anti-CD43, mouse anti-CD45, mouse anti-CD45RO, mouse antiCD56, rabbit anti-CD79a, rabbit anti-Cytokeratin 7 and AE1/AE3/PCK26 anti-Pan keratin cocktail (all from Ventana, Tucson, AZ, USA), in addition to mouse antiCD2 and mouse anti-CD138/syndecan-1 (from Cell Marque, Rocklin, CA, USA). By immunohistochemistry, the large cells were strongly positive for CD30 and also expressed CD2, CD43, CD45, and CD45RO. The large cells were partially positive for CD4 and CD5 while they were negative for expression of CD3, ALK, CD56, CD15, CD20, CD79a, CD138, pan-keratin and cytokeratin 7. A diagnosis of anaplastic large cell lymphoma, ALK-negative was rendered.

Staging workup included a whole body positron emission tomography/computed tomography (PET/CT), which showed numerous normal sized but mildly hypermetabolic lymph nodes scattered throughout the bilateral cervical, axillary, mediastinal, hilar, external iliac and inguinal lymph node chains, along with foci of abnormal metabolic activity in spleen, upper abdominal mesentery, gastrocolic ligament and subpleural consolidation. However, no areas of abnormal hypermetabolism were seen in the rectum. Bilateral bone marrow biopsies showed a mildly hypercellular marrow with trilineage hematopoiesis with no involvement by the lymphoma. Serum LDH level was increased $361 \mathrm{IU} / \mathrm{L}$. B-2 microglobulin was also elevated at $4.87 \mathrm{ug} / \mathrm{L}$. Patient was stratified under high risk group by International Prognostic Index (IPI) score of 4 . The patient has been advised CHOP chemotherapy regimen followed by consolidation with autologous stem cell transplantation in first remission. This case represents the first case of ALCL, ALK-primarily diagnosed in the colon as a rectal ulcer.

\section{Discussion}

ALCL, ALK - is included as a provisional entity in the WHO classification, and is defined as a CD30+ T-cell neoplasm that is not reproducibly distinguishable on morphological grounds from ALCL, ALK+, but lacks ALK protein. Absent expression of ALK has been demonstrated to portend a significantly worse prognosis with a 5 -year overall survival of $37 \%$, as compared to with expression of ALK [2]. The peak incidence of ALCL, ALK - is in adults (40 - 65 years) unlike ALCL, ALK+ which occurs mostly in children and young adults. The index case had a slight younger age at presentation (37year-old male). ALCL, ALK- typically presents with lymph node involvement, with extranodal disease being less prevalent than in ALK+ cases. The most common sites of extranodal involvement are the skin, bone and soft tissues. The presenting feature of the index case is noteworthy for the rectum being the extranodal site of 
involvement and for the subtle finding of a small aphthous ulcer, not suspicious of lymphoma. Rectum has never been reported as the primary site of diagnosis for ALCL, ALK negative.

Lymphomas of the gastrointestinal tract (GIT) account for $4 \%-20 \%$ of all NHL and their incidence has been steadily increasing [3]. The stomach is by far the most common site where up to $75 \%$ of all GI lymphomas occur, while lymphoma accounts for only $25 \%$ of smallintestinal tumors and less than $0.5 \%$ of colorectal neoplasms [4]. The vast majority of GIT lymphomas are of B-cell lineage with low grade extranodal mucosaassociated lymphoma (MALT) being most common, followed by diffuse large B-cell lymphoma, Burkitt lymphoma and mantle cell lymphoma, the later often presenting as lymphomatoid polyposis [5]. T-cell lymphomas of the GI tract are much less common, though specific clinicopathologic entities have been described in the GIT, such as the enteropathy-associated T-cell lymphoma (EATL) and the NK-cell enteropathy [6], both of which can involve small intestine and colon. Rare cases of ALCL have been documented in the esophagus [7,8], stomach [9-11], and in the pancreas [12,13]. In addition, ALCL was identified in one case at the site of ileocolonic anastomosis in a patient treated for colonic adenocarcinoma [14], and it also has been described in the setting of ulcerative colitis [15]. An autopsy-based study of 5 cases of systemic ALCL described involvement of GIT in 3 subjects, including esophagus, small bowel, gallbladder, and submucosa of the colon [16]. All three patients had systemic disease. ALCL is treated with chemotherapy, and an extensive surgery of the gastrointestinal site would negatively affect the prognosis. Therefore, the common challenge in such cases is to avoid misinterpretation of ALCL as a poorly differentiated adenocarcinoma.

Microscopically, ALCL is characterized by large lymphoid cells with pleomorphic nuclei and abundant cytoplasm. As morphologic features are often subjective and there are no unique defining immunophenotypic or genetic features, ALCL, ALK- can be a difficult diagnosis. The absent ALK expression differentiates our case from ALCL, ALK positive. Additional differential diagnosis of a lymphoma with large pleomorphic cells, abundant cytoplasm and strong CD30 expression may include CD30 positive diffuse large B-cell lymphoma, peripheral T-cell lymphoma, not otherwise specified (PTCL-NOS), EATL, syncytial variant of classical Hodgkin lymphoma, and NK-cell enteropathy. The absent B-cell markers CD20 and CD79a help to exclude a B-cell lymphoma. The anaplastic morphology and the strong membranous and Golgi-associated staining pattern of $\mathrm{CD} 30$ are the most important features to differentiate our case from
PTCL-NOS. The International Peripheral T-Cell Lymphoma Project documented inferior survival for patients with PTCL-NOS in comparison to ALCL, ALK-, highlighting the importance of correct differential diagnosis in between these two diseases. EATL is an important entity to consider, since it can involve colon, and occasionally, may exhibit highly pleomorphic morphologic features and CD30 positivity. The most important finding to exclude this entity is the lack of small intestinal mass by upper endoscopy and the absent history of celiac disease. The syncytial variant of classical Hodgkin lymphoma could present with similarly large cells and CD30 positivity; however, the lack of eosinophils and mixed inflammatory milieu in the background, the expression of CD43 and partial CD45 by the large cells and the lack of CD15 in our case, are all against classical Hodgkin lymphoma. Finally, an NK/T-cell lymphoma, or the so-called NK-cell enteropathy, a recently recognized benign lymphoproliferative disease mimicking intestinal lymphoma that may involve colon, express CD56, which was negative in our case.

Another notable feature of our case is the history of RCC in this patient six years prior the diagnosis of ALCL, ALK negative. In a previous study on the association between renal cell carcinoma (RCC) and nonHodgkin lymphoma (NHL), two patients were seen in whom RCC preceded NHL, similar to our case [17]. One patient (aged 67 years) was symptomatic, while NHL was an incidental finding in the second patient (aged 69 years). Both the patients had diffuse large B cell lymphoma. The study showed an increased risk of RCC following a previous NHL diagnosis but did not demonstrate increased risk of NHL in patients with a previous RCC diagnosis, possibly due to small number of cases [17]. A common genetic or environmental factor may be involved in the concurrent evolution of the two malignancies. Since currently no known pathological link between the two malignancies is known at the present time, the predisposing features for these two different malignant neoplasms in the same patient are yet to be explored.

In summary, we describe a rare extranodal manifestation of systemic ALCL, ALK- in the rectum. In this case the primary diagnosis was established based on a colonoscopic biopsy of a small rectal aphthous ulcer. This report highlights the differential diagnosis and the diagnostic pitfalls in CD30 positive T-cell lymphomas that may involve the colon. Correct diagnosis is facilitated by a careful immunophenotypic analysis and is essential for delivering the appropriate therapy, avoiding an unnecessary gastrointestinal resection and opening the possibility for lymphoma-directed chemotherapy. 


\section{REFERENCES}

[1] D. Y. Mason, N. L. Harris, G. Delsol, H. Stein, E. Campo, Kinney, et al., "Anaplastic Large Cell Lymphoma, ALKNegative," In: S. H. Swerdlow, E. Campo, N. L. Harris, E. S. Jaffe, S. A. Pileri, H. Stein, J. Thiele and J. W. Vardiman, Eds., WHO Classification of Tumours of Haematopoietic and Lymphoid Tissues, International Agency for Research on Cancer, Lyon, 2008, pp. 317-319.

[2] K. J. Savage, N. L. Harris, J. M. Vose, F. Ullrich, E. S. Jaffe, J. M. Connors, et al., "ALK-Anaplastic large-Cell Lymphoma Is Clinically and Immunophenotypically Different from both ALK+ ALCL and Peripheral T-Cell Lymphoma, Not Otherwise Specified: Report from the International Peripheral T-Cell Lymphoma Project," Blood, Vol. 111, No. 12, 2008, pp. 5496-5504. doi:10.1182/blood-2008-01-134270

[3] R. Otter, R. Bieger, P. M. Kluin, J. Hermans and R. Willemze, "Primary Gastrointestinal Non-Hodgkin's Lymphoma in a Population-Based Registry," British Journal of Cancer, Vol. 60, No. 5, 1989, pp. 745-750. doi:10.1038/bjc. 1989.351

[4] S. Nakamura, T. Matsumoto, M. Iida, T. Yao and M. Tsuneyoshi, "Primary Gastrointestinal Lymphoma in Japan: A Clinicopathologic Analysis of 455 Patients with Special Reference to Its Time Trends," Cancer, Vol. 97, No. 10, 2003, pp. 2462-2473. doi:10.1002/cncr.11415

[5] F. d'Amore, H. Brincker, K. Gronbaek, K. Thorling, M. Pedersen, M. K. Jensen, et al., "Non-Hodgkin's Lymphoma of the Gastrointestinal Tract: A Population-Based Analysis of Incidence, Geographic Distribution, Clinicopathologic Presentation Features, and Prognosis. Danish Lymphoma Study Group," Journal of Clinical Oncology, Vol. 12, No. 8, 1994, pp. 1673-1684.

[6] A. Mansoor, S. Pittaluga, P. L. Beck, W. H. Wilson, J. A. Ferry and E. S. Jaffe, "NK-Cell Enteropathy: A Benign NK-Cell Lymphoproliferative Disease Mimicking Intestinal Lymphoma: Clinicopathologic Features and Follow-Up in a Unique Case Series," Blood, Vol. 117, No. 5, 2011, pp. 1447-1452. doi:10.1182/blood-2010-08-302737

[7] C. W. Ross, C. A. Hanson and B. Schnitzer, "CD30 (Ki-1)-Positive, Anaplastic Large Cell Lymphoma Mimicking Gastrointestinal Carcinoma," Cancer, Vol. 70, No. 10, 1992, pp. 2517-2523. doi:10.1002/1097-0142(19921115)70:10<2517::AID-CN CR2820701021>3.0.CO;2-U

[8] N. Wu, L. Pang, Z. Chen, Y. Wang, Q. Ma, G. Chen, et al., "Primary Esophageal CD30-Positive ALK-Positive Anaplastic Large Cell Lymphoma: A Case Report and Literature Review," Journal of Gastrointestinal Cancer, Vol. 42, No. 1, 2011, pp. 57-60.
[9] M. Paulli, R. Rosso, S. Kindl, E. Boveri E. , E. Bonoldi, V. Stracca, et al., "Primary Gastric CD30 (Ki-1)-Positive Large Cell Non-Hodgkin's Lymphomas. A Clinicopathologic Analysis of Six Cases," Cancer, Vol. 73, No. 3, 1994, pp. 541-549. doi:10.1002/1097-0142(19940201)73:3<541::AID-CNCR 2820730308>3.0.CO;2-1

[10] H. Matsumoto, H. Koga, K. Honda, Y. Sadahira, Y. Suetugu, M. Mikami, et al., "Characterization of Secondary GI Lesions with Anaplastic Large-Cell (Ki-1) Lymphoma: A First Report of Two Cases," Gastrointestinal Endoscopy, Vol. 61, No. 4, 2005, pp. 607-609. doi:10.1016/S0016-5107(04)02785-3

[11] M. Sugimoto, M. Kajimura, H. Hanai, N. Shirai, F. Tanioka and E. Kaneko, "G-CSF-Producing Gastric Anaplastic Large Cell Lymphoma Complicating Esophageal Cancer," Digestive Diseases and Sciences, Vol. 44, No. 10, 1999, pp. 2035-2038. doi:10.1023/A:1026674402695

[12] C. S. Chim, J. Ho, G. C. Ooi, C. Choy and R. Liang, "Primary Anaplastic Large Cell Lymphoma of the Pancreas," Leukemia \& Lymphoma, Vol. 46, No. 3, 2005, pp. 457-459. doi:10.1080/1042819040007474

[13] Y. Cohen, D. Libster, G. Amir, N. Hiller, N. Da'as, D. Ben Yehuda, et al., "Primary ALK Positive Anaplastic Large Cell Lymphoma of the Pancreas," Leukemia \& Lymphoma, Vol. 44, No. 1, 2003, pp. 205-207. doi:10.1080/1042819021000054715

[14] M. R. Cooperberg and P. N. Fiedler, "Ki-1 Anaplastic Large-Cell Lymphoma Occurring at the Site of Ileocolonic Anastomosis in a Patient Treated Surgically for Colonic Adenocarcinoma: Case Report and Review of the Literature," Annals of Diagnostic Pathology, Vol. 5, No. 3, 2001, pp. 162-167. doi:10.1053/adpa.2001.25408

[15] L. A. Ashworth, A. Billett, P. Mitchell, F. Nuti, C. Siegel and A. Bousvaros, "Lymphoma Risk in Children and Young Adults with Inflammatory Bowel Disease: Analysis of a Large Single-Center Cohort," Inflammatory Bowel Diseases, Vol. 18, No. 5, 2012, pp. 838-843. doi:10.1002/ibd.21844

[16] M. B. Mosunjac, J. B. Sundstrom and M. I. Mosunjac, "Unusual Presentation of Anaplastic Large Cell Lymphoma with Clinical Course Mimicking Fever of Unknown Origin and Sepsis: Autopsy Study of Five Cases," Croatian Medical Journal, Vol. 49, No. 5, 2008, pp. 660-668. doi: $10.3325 / \mathrm{cmj} .2008 .5 .660$

[17] C. Lossos, A. Ferrell, R. Duncan and I. S. Lossos, "Association between Non-Hodgkin Lymphoma and Renal Cell Carcinoma," Leukemia \& Lymphoma, Vol. 52, No. 12, 2011, pp. 2254-2261. doi: $10.3109 / 10428194.2011 .603443$ 